\title{
In Silico discovery of transcription factors as potential diagnostic biomarkers of ovarian cancer
}

\author{
Mandeep Kaur', Cameron R MacPherson', Sebastian Schmeier ${ }^{1}$, Kothandaraman Narasimhan², Mahesh Choolani ${ }^{3}$ \\ and Vladimir B Bajic ${ }^{1 *}$
}

\begin{abstract}
Background: Our study focuses on identifying potential biomarkers for diagnosis and early detection of ovarian cancer (OC) through the study of transcription regulation of genes affected by estrogen hormone.

Results: The results are based on a set of 323 experimentally validated OC-associated genes compiled from several databases, and their subset controlled by estrogen. For these two gene sets we computationally determined transcription factors (TFs) that putatively regulate transcription initiation. We ranked these TFs based on the number of genes they are likely to control. In this way, we selected 17 top-ranked TFs as potential key regulators and thus possible biomarkers for a set of 323 OC-associated genes. For 77 estrogen controlled genes from this set we identified three unique TFs as potential biomarkers.

Conclusions: We introduced a new methodology to identify potential diagnostic biomarkers for OC. This report is the first bioinformatics study that explores multiple transcriptional regulators of OC-associated genes as potential diagnostic biomarkers in connection with estrogen responsiveness. We show that $64 \%$ of TF biomarkers identified in our study are validated based on real-time data from microarray expression studies. As an illustration, our method could identify CP2 that in combination with CA125 has been reported to be sensitive in diagnosing ovarian tumors.
\end{abstract}

\section{Background}

Ovarian cancer $(\mathrm{OC})$ is the leading cause of death among gynecological malignancies and represents the fifth leading cause of cancer-related deaths in women. The disease is diagnosed at stage when cancer has already metastasized beyond the ovary in approximately $70 \%$ of patients and only $30 \%$ of these patients with this advanced-stage OC survive 5 years after initial diagnosis [1]. This inability to detect ovarian carcinoma during the early organ-confined stage combined with the lack of effective therapies for advanced-stage disease contributes to lethal effects of this cancer. In patients with metastasized $\mathrm{OC}$, most relapse and ultimately die due to the development of drug resistance [2].

Early diagnosis greatly enhances the chances of successful cancer treatment. To this date, very few earlydetection approaches have shown promise for routine

\footnotetext{
* Correspondence: vladimir.bajic@kaust.edu.sa

'Computational Bioscience Research Center, King Abdullah University of Science and Technology, Thuwal 23955-6900, Kingdom of Saudi Arabia Full list of author information is available at the end of the article
}

clinical use. The most commonly used marker of OC is CA125, but it is expressed in $50-60 \%$ of patients during early stages of the disease [3]. Several biomarkers either individually or in combination with CA 125 have been proposed for early-detection and screening of OC [4]. FDA recently cleared an In Vitro Diagnostic Multivariate Index Assay (IVDMIA) i.e. OVA1 test that involves analysis of five serum biomarkers for assessing ovarian cancer risk in women [5]. Over the past few years it has become increasingly evident that many molecular changes observed in cancer cells involve deregulation of gene expression. Understanding the underlying molecular mechanisms of gene regulation could thus be crucial for identifying the key genes or proteins that can be exploited as prognostic or diagnostic biomarkers in OC. This makes transcription factors (TFs) an interesting target for further exploration in this direction [6]. The majority of oncogenic signaling pathways converge on sets of TFs that ultimately control gene expression patterns characteristic for tumor formation and progression, as well as metastasis. Since many of these TFs are
C Biomed Central

(c) 2011 Kaur et al; licensee BioMed Central Ltd. This is an Open Access article distributed under the terms of the Creative Commons Attribution License (http://creativecommons.org/licenses/by/2.0), which permits unrestricted use, distribution, and reproduction in any medium, provided the original work is properly cited. 
inactive in the cancer affected tissues under normal physiological conditions and their expression and activities are tightly regulated, these TFs represent highly desirable and logical points of therapeutic interference in cancer development, progression and prognostication [7-9], markers for cancer [10], potential prognostic markers $[7,11]$ and targets for drug therapy [12]. More recently the use of TFs as markers for the disease itself has been reported and they have been detected in the blood [10,13,14]. Another study [7] investigated the role of survival-related profile, pathways, and TFs in OC. The study reported that 13 out of 111 TFs were associated with overall survival in patients with OC. Since hormones also play an important role in gene expression [15] and are implicated in many cancers [16-19], it also becomes important to study the effects of hormones in cancers. It is documented that high levels of expression of estrogen receptor alpha $(E R \alpha)$ has been observed in many OCs and OC cells are growth responsive to both estrogen and anti-estrogens [20]. This emphasizes that $\mathrm{ER} \alpha$ could have therapeutic potential for at least a sub-group of OC patients [21-23]. The hormone replacement therapy has also been linked with an increased risk of OC [24]. Recently, the prognostic value of estrogen receptors (ERs) for OC has been emphasized [25]. It is worth noting that the studies explaining the role of hormones in $\mathrm{OC}$ are few and require elaborative investigations [26]. Therefore, in the present study we focus on transcription regulation and also estrogen control of genes in OC. The question we addressed is to identify potential diagnostic TFs implicated in regulating the expression of $\mathrm{OC}$ genes that in turn could potentially be regulated by estrogen hormone. We aimed at linking hormone induction to overall transcription regulation of genes in cancer cells. We anticipate that the target pool of biomarkers could be revealed by studying specific signatures within the hormone dependent regulatory gene networks.

Computational approaches are a pragmatic and inexpensive way to identify the key regulatory genes, hence, pinpointing the targets for experimental validation. For example, bioinformatics methods were employed to identify candidate genes for discriminating different tumoral histotypes for OC, lung and breast cancer diagnostics [27]. In general, steroid hormones are recognized initially by hormone receptors that then bind to Hormone-Response Elements (HREs) on DNA and control the expression of some of their target genes. This can be used to predict a part of genes that are potentially controlled by hormone receptors under the condition that promoters of these genes contain HREs. Thus, the identification and characterization of HREs is critical for our understanding of hormone driven gene expression and regulation in various cancers and computational methods could be of great help [28-31]. To contribute to the discovery of diagnostic biomarkers for $\mathrm{OC}$ we developed a computational method which, in combination with the manual curation of the literature information, helped us to identify the potential biomarkers that could regulate a set of genes implicated in OC. Our analysis singles out several such TFs. The previous efforts that used the bioinformatics approach to identify a TF, E2F5, and then experimentally confirm it as a potential diagnostic biomarker for $\mathrm{OC}$ were made by our group [6]. Our current study is, to the best of our knowledge, the only one that targets through purely bioinformatics approach, transcriptional regulators of $\mathrm{OC}$-associated genes as potential biomarkers in relation to estrogen responsiveness.

\section{Results and Discussion}

This study identified potential biomarkers important for overall transcriptional regulation of $\mathrm{OC}$ genes and specifically for a sub-group of $\mathrm{OC}$ genes controlled by estrogen. To identify genes controlled by estrogen, two approaches were used: (a) prediction of estrogen response elements (EREs) on the promoters of OC genes, and (b) finding the experimental evidence for estrogen control in published databases. The presence of EREs in a promoter suggests that ERE sites may be used by activated hormone receptors and consequently could affect gene expression. The EREs were predicted on 246 promoters corresponding to 65 genes. Out of these 65 genes, 11 genes had experimental evidence of being responsive to estrogen as these genes were found in either KBERG [32] or ERTargetDB [33] databases. In the dataset, there were 258 genes lacking predicted EREs in their promoters. Out of these 258 genes, 66 were under estrogen control as supported by KBERG or ERTargetDB. We split the genes into four groups that were analyzed further. These four groups were:

Group 1) genes with predicted EREs and with experimental evidence of estrogen responsiveness (11 genes);

Group 2) genes with predicted EREs with no experimental evidence of estrogen responsiveness ( 54 genes);

Group 3) genes without ERE predictions but with experimental evidence of estrogen responsiveness (66 genes); and

Group 4) genes without ERE predictions and no experimental evidence of estrogen responsiveness (192 genes).

It is to be emphasized here that ERs can also affect gene expression by forming protein-protein complexes with other TFs such as activator protein-1 (AP-1), Sp1 family TFs, nerve factor- $\beta(N F-\beta)$, etc. These complexes in turn can bind to the genes' promoters and regulate gene expression. Through these ERE-independent pathways, ERs can control the expression of many genes, 
making them estrogen-responsive, but without involvement of the full ERE. Therefore, the EREs, though good indicators that genes may be controlled by estrogen, are not essential for responsiveness to estrogen. On its own, the presence of EREs in the promoter region of a gene is not conclusive evidence of the hormonal control of expression of that gene. However, the presence of EREs in a promoter suggests that such ERE sites could be used by activated hormone receptors and consequently could affect gene expression.

\section{(a) Functional analysis of target genes}

The GO analysis was performed as described in Material and Methods. The results for each group are discussed below:

Group 1: This group contains genes with predicted EREs and with experimental evidence of estrogen responsiveness. This group contains only 11 genes (3\% of total 323 genes under study). The GO analyses of these genes show that $45 \%$ of genes in group 1 had kinase activity (additional file 1).

Group 2: The genes in this group (17\% of 323 genes) have predicted EREs but they lack experimental evidence of being responsive to estrogen. It may be assumed that these genes might be under the control of estrogen hormone but this observation has not been conclusively demonstrated or supported by literature. GO analyses revealed that 24\% (13 out of 54) had kinase activity and $26 \%$ of genes were involved in nucleotide binding (additional file 1).

Group 3: All the 66 genes (20\%) in this group have no predicted EREs but have experimental evidence of being estrogen responsive. The genes in this group were mainly involved in organ development (32\%), positive regulation of cellular processes $(29 \%)$ and intracellular signaling cascade (26\%). Only three (5\%) were involved in regulation of I-kappaB kinase/NF-kappaB cascade (additional file 1).

Group 4: The genes in this group contain no ERE predictions, and have no experimental evidence in support of an estrogen response and constitute approximately 60\% (192 of 323) of the total number of genes under study. Most of these genes $(38 \%)$ were involved in cellular protein metabolic process. Some of the genes in this group (23\%) were part of cell surface receptor linked signal transduction, while others (21\%) had kinase activity (additional file 1).

\section{(b) Analysis of TF binding sites (TFBSs)}

The gene groups were then subjected to TFBS analysis as explained in Materials and Methods. The results of TFBS analysis are summarized in following sections:

(i) Distribution of TFBSs in promoters of OC genes

We predicted 9246 TFBSs corresponding to 299 TFs using 522 TRANSFAC matrices. The distribution of all predicted TFBSs in OC genes is presented in Figure 1. This figure shows the distribution of TFBSs using the Gaussian-kernel density estimator implemented in the $\mathrm{R}$ statistical environment under the function name, "plot. density"; the greatest density is just upstream of transcription start sites (TSSs).

\section{(ii) Distribution of TFBSs and EREs in promoters of genes that contain predicted EREs}

It has been demonstrated in the literature [34] that distance between ERE and TFBS is crucial for transcription of the gene. Some studies also suggest that most of the ERE effects are at very large distance from TSSs of genes they regulate [35]. The activities of the downstream promoter of vitellogenin gene A1 are shown to be controlled by EREs located 330 bases upstream of the TSS and it is speculated that EREs located up to 1.5 $\mathrm{kb}$ downstream can also control the promoter [36]. The distribution of distances between TFBSs and EREs within the promoters of $\mathrm{OC}$ genes that have predicted EREs is presented in Figure 2. For this analysis we pooled the results of group 1 and group 3 genes as both these groups had genes experimentally validated to be estrogen responsive. We found 260 TFs had TFBSs completely overlapping with EREs, whereas 299 TFs had TFBSs predicted at a maximum distance of 95 bases from predicted EREs. The detailed results are provided (additional file 2). Most of the TFs having TFBSs within a distance of 95 bases from predicted EREs were involved in cancer related biological processes. This suggests that the closeness of ERE to some other TFBSs implicated in our analysis could synergistically function in $\mathrm{OC}$.

\section{(iii) Identification of TFs unique to groups of genes}

The enriched TFBSs (i.e. ORI $>=2$, Materials and Methods) were used for network construction. The TFs potentially regulating maximum number of genes in each group were identified. The TFs were ranked based on the number of genes they regulate as demonstrated via TFBSs on the promoters of the genes. We used a cut-off of $80^{\text {th }}$ percentile to identify the TFs regulating maximum number of genes in a group. Any TF having TFBSs in genes below $80^{\text {th }}$ percentile were not included in further analysis. These steps lead us to identify TFs unique to sets of genes in each group (Figure 3). Figure 3 also show that multiple TFs may regulate set of genes in different groups thus highlighting the TF families that could be of relevance for further investigations in OC.

Using the cut-off as mentioned above, we identified 18 TFs having TFBSs in promoters of 11 genes in group 1. The 54 genes in group 2 contained TFBSs for 31 TFs. In group 3, we found TFBSs for 23 TFs in promoters of 66 genes. For group 4, we identified TFBSs for 31 TFs in promoters of 192 genes. We then combined groups 1 and 3 because the genes in these groups were found 


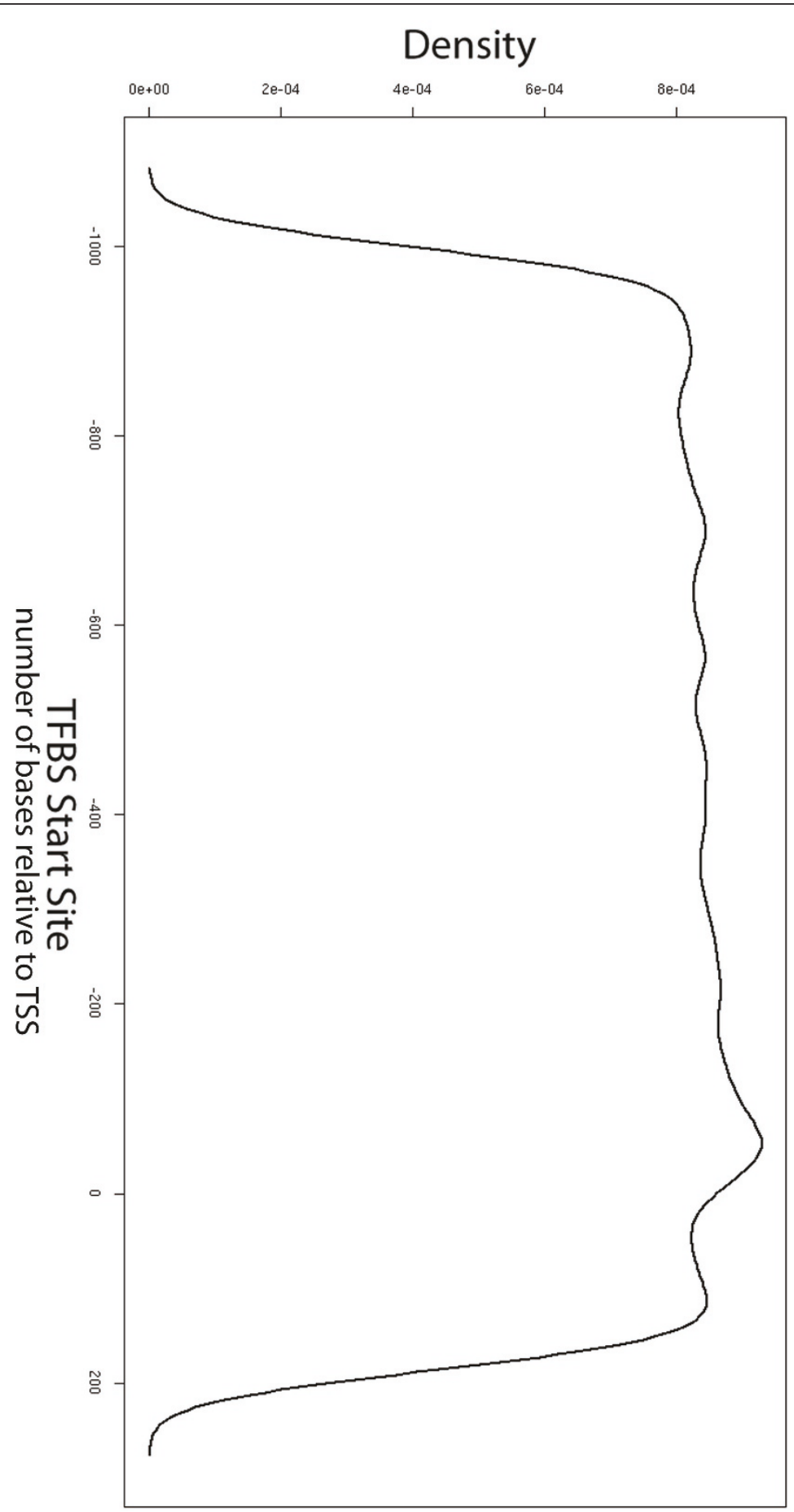

Figure 1 Distribution of all predicted TFBSs in OC genes. X-axis: distance in nucleotides from TFBS relative to TSS. Y-axis: estimated density of TFBSs as calculated by a Gaussian-kernel density function implemented in the R-statistical environment as "plot.density"; the distances of all TFBSs from the TSS are distributed across 512 points and convolved using a Gaussian kernel.

experimentally to be controlled by estrogen. This new group we refer to as 'experimentally controlled genes'. We found that there were 17 TFs common to both gene groups 1 and 3 , with only one TF unique to group 1 and six TFs unique to group 3 (additional file 3). Thus, we found 24 TFs that have TFBSs on the promoters of experimentally controlled genes only. The comparison of these 24 TFs with group 2 (group of genes containing 


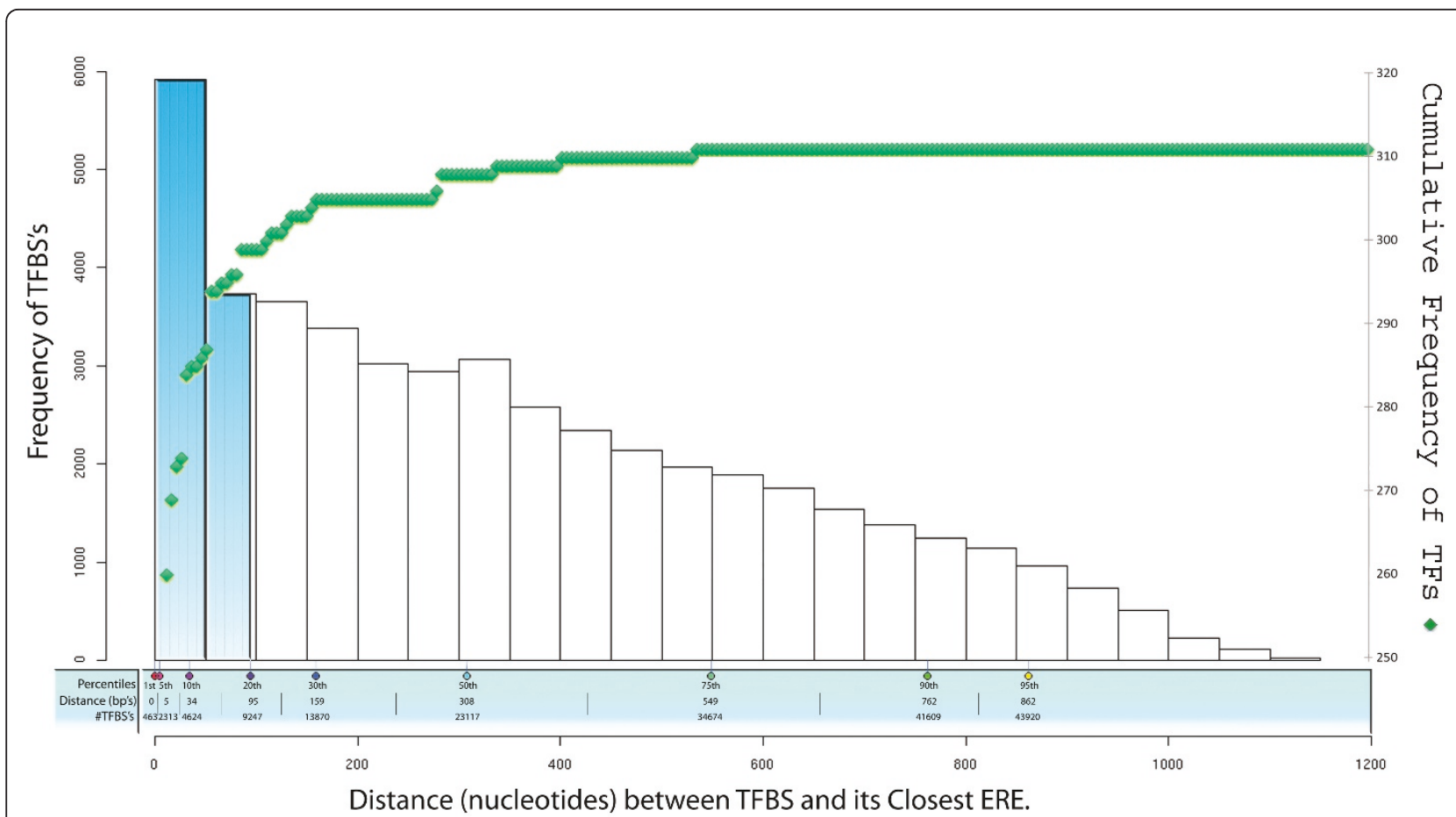

Figure 2 Histogram and cumulative plot of distances between TFBSs and EREs in OC promoters. X-axis: distance in nucleotides between TFBSs and its' closest ERE; maximum distance is limited to the size of promoters (1200 nt); frequencies of TFBSs for selected percentiles are given. Y-axis (left, histogram): frequency of TFBSs per 50 nucleotides (24 bins); the 20th percentile is highlight blue for convenience. Y-axis (right, dot-plot): cumulative frequency of TFBSs.

predicted EREs but lacking experimental evidence of control by estrogen) revealed that there were 19 TFs common with the experimentally controlled genes, and 12 TFs unique to group 2 (additional file 4). Similar comparison of TFs for experimentally controlled genes with group 4 revealed 20 common TFs and 11 unique TFs for group 4 (additional file 5).

The above analysis suggests that combinations of TFs could regulate different sets of genes (Figure 3). We propose that the genes in group 2 can be considered as new targets for experimental evaluation of estrogen control. This proposition is based on the presence of predicted EREs in promoters of these genes. The presence of EREs in promoters of genes does not establish that the genes are under estrogen control, however, the ERE predictions open a possibility that these genes may be under such a control. (iv) Identification of potential markers for 323 OC genes We propose that the TFs regulating a maximum number of $\mathrm{OC}$ genes can be regarded as potential candidates for biomarkers for OC. This is based on the concept that such TFs are the drivers of the potential deregulation of genes in $\mathrm{OC}$, and thus are relevant for use as biomarkers. To identify potential candidates, we ranked TFs with regards to the number of genes that have TFBSs for a particular TF (see additional file 6 for list of all ranked TFs). The ranking produced a list of $17 \mathrm{TFs}$
(Table 1 ) each potentially regulating more than 200 OC genes. Top ranked three TFs (entrez ID 5079, 7020 and 1385) had TFBSs in promoters of 289, 268 and 255 genes, respectively. The top ranked TF (PAX-5) belongs to a family of paired box TFs. The protein product of this gene is a B-cell lineage specific activator and has been identified as a marker for the discrimination of low- to intermediate-grade pulmonary neuroendocrine carcinomas from high-grade with $100 \%$ specificity and $79 \%$ sensitivity in surgical specimens [37] and for diagnosis of undifferentiated malignant neoplasms [38]. The TF ranked at position two in the list (entrez ID 7020) is AP-2 and regulates the expression of amyloid precursor protein in oral squamous cell carcinomas [39]. It has also been linked with breast cancer [40]. The third ranked TF (entrez ID 7546) is zinc finger protein (ZIC2) and has been found to be differentially expressed in endometrial cancers with lymph node metastasis [41] and has even been patented as a small-cell lung cancer associated antigen (US Patent 7314721, issued on January 1, 2008, http://www.patentstorm.us/patents/ $7314721 /$ fulltext.html.

\section{(v) Identification of potential markers for estrogen regulated genes}

After identifying TFs potentially regulating a maximum number of OC genes, we looked for TFs uniquely 


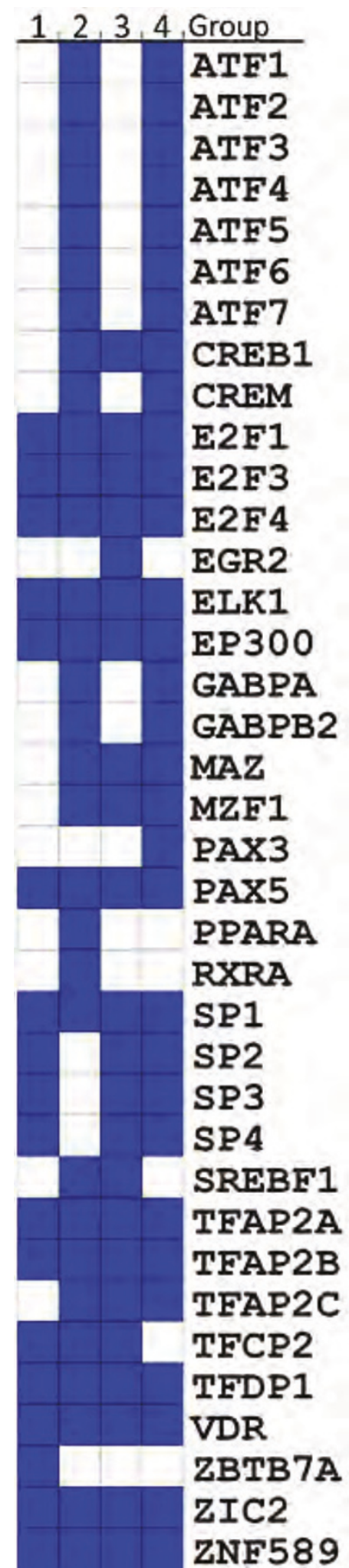

Figure 3 Heat-map showing combinations of TFs regulating different sets of genes. Blue blocks across the groups (columns) indicate that several TFs (rows) may potentially regulate genes in different groups. regulating estrogen controlled genes in groups 1 and 3 . For the 'experimentally controlled genes', we identified 17 top ranked TFs (Table 1). This group has three unique TFs ranked 15, 16 and 17, while others were common with the previous ranked list of TFs (for all categories combined). The TF ranked at position 15 is CP2 (entrez ID 7024), which has been targeted as a diagnostic marker for ovarian carcinoma [42]. The combination of CA125 with CP2 has been reported to be sensitive in diagnosing non-mucinous ovarian tumors [43]. The TFs ranked 16 and 17 in the list are Sp2 (entrez ID 668) and Sp4 (entrez ID 6671), respectively. The PubMed search using keywords "Sp4 ovarian cancer" retrieved only one abstract related to the study that tested new analogues of a natural antibiotic 'Mithramycin' as inhibitors of Sp1-dependent transcription in OC xenografts [44]. Therefore, we propose that Sp4 can be investigated as a potential new gene or marker for OC. Another observation is that the family of Sp TFs might have an important role to play in OC. Sp3 and Sp4 are involved in estrogen mediated gene expression in MCF7 breast cancer cells [45]. Sp3 (entrez ID 6670) has been shown to control the expression of PTEN (phosphatase and tensin homolog deleted on chromosome 10) in ovarian carcinomas [46]. The proximal binding of Sp1 to the TSS has been demonstrated to be a requirement for positive interactions with the ER to enhance transcription and also to stabilize weak interactions of ER [47]. Three TFs (TFCP2, SP2 and SP4) unique to estrogen controlled group can be tested as specific biomarkers for the sub-group of patients that are influenced by estrogen hormones.

The analysis presented above has enabled us to identify potential biomarkers that putatively regulate transcription of a group of OC-associated genes. The results presented here can be justified by the fact that the analysis could identify some of the known biomarkers (currently under investigation), such as CP2 and ZIC2. The study has identified unique potential biomarkers for a sub-group of genes that are known to be under estrogen control.

\section{(c) Preliminary validation of biomarkers using publically} available data

To see if the TFs identified in the current study have functional roles and the potential to be used as diagnostic biomarkers during early and advanced stages of OC, we looked at genes specifically (over-expressed) as represented in microarray analysis at different stages of ovarian serous adenocarcinoma and metastatic ovarian serous papillary adenocarcinoma tissue samples [48]. For identifying the genes and potential TFs associated with these genes we used data from 24 non-metastatic ovarian cancer tissue samples against 3 metastatic 
Table 1 Rankings of TFs (potential biomarkers) for complete gene set of 323 OC genes and for the 'experimental group'

\begin{tabular}{|c|c|c|c|c|c|c|c|}
\hline \multicolumn{4}{|c|}{ Ranked TFs for 323 genes } & \multicolumn{4}{|c|}{ Ranked TFs for Experimental group } \\
\hline TF_ID & TF_symbol & $\begin{array}{c}\text { Number of Gene Targets } \\
\text { (out-degree) }\end{array}$ & Ranks & TF_ID & TF_symbol & $\begin{array}{c}\text { Number of Gene Targets } \\
\text { (out-degree) }\end{array}$ & Ranks \\
\hline 5079 & PAX5 & 289 & 1 & 5079 & PAX5 & 68 & 1 \\
\hline 7020 & TFAP2A & 268 & 2 & 7020 & TFAP2A & 60 & 2 \\
\hline 1385 & CREB1 & 255 & 3 & 7546 & $\mathrm{ZIC2}$ & 60 & 3 \\
\hline 7546 & $\mathrm{ZIC2}$ & 253 & 4 & 1869 & E2F1 & 57 & 4 \\
\hline 7421 & VDR & 249 & 5 & 51385 & ZNF589 & 55 & 5 \\
\hline 1869 & E2F1 & 248 & 6 & 7027 & TFDP1 & 54 & 6 \\
\hline 7022 & TFAP2G & 246 & 7 & 1871 & E2F3 & 54 & 7 \\
\hline 7027 & TFDP1 & 245 & 8 & 7421 & VDR & 54 & 8 \\
\hline 1874 & E2F4 & 245 & 9 & 1874 & E2F4 & 54 & 9 \\
\hline 1871 & E2F3 & 245 & 10 & 7021 & TFAP2B & 52 & 10 \\
\hline 7021 & TFAP2B & 242 & 11 & 6670 & SP3 & 52 & 11 \\
\hline 2033 & EP300 & 223 & 12 & 2033 & EP300 & 52 & 12 \\
\hline 6670 & SP3 & 222 & 13 & 6667 & SP1 & 48 & 13 \\
\hline 6667 & SP1 & 216 & 14 & 2002 & ELK1 & 48 & 14 \\
\hline 51385 & ZNF589 & 216 & 15 & 7024 & TFCP2 & 42 & 15 \\
\hline 7593 & ZNF42 & 214 & 16 & 6668 & $S P 2$ & 40 & 16 \\
\hline 2002 & ELK1 & 210 & 17 & 6671 & SP4 & 40 & 17 \\
\hline
\end{tabular}

TFs unique to both groups are shown in bold italics.

ovarian cancer tissue samples (obtained from microarray gene expression database) CleanEx (Microarray database) http://www.cleanex.isb-sib.ch/index.html[49] using dataset number AFFY004 [50]. The primary data was used in dataset number AFFY004 was taken from [48] and the details of primary data have been summarized (additional file 7). We could identify three TFs (ZIC2, $E 2 F 4$ and TFCP2) in this dataset of over-expressed genes in OC. TFs such as E2F1, E2F3, TFAP2A, VDR, TFAP2G, SP3, ZNF42, CREB1 and ELK1 were found to be over-expressed by $>=1.5$ folds in OC datasets analyzed in Oncomine http://www.oncomine.org/[51]. Our study has shown that $65 \%$ of the TFs regulating genes in a full set of 323 OC genes and $47 \%$ of the TFs regulating estrogen controlled sub-set of genes of our predication set could be identified in real-time data from published microarray expression studies (Table 2). This analysis shows that the expression of a number of the TFs identified in the present study are affected in OC and these TFs may have the potential to be used as biomarkers. By definition, a biomarker is useful if it can detect the disease in early stages in a patient as compared to a healthy individual. To study this, we performed an analysis based on the expression profiles available in Oncomine targeting 'Ovarian serous adenocarcinoma' which is the most common form of the OC [52-54]. We could identify TFAP2A, VDR, TFAP2G and $E 2 F 3$ as over-expressing genes in at least one of the three datasets we studied (Table 3). The expression of CA125 (a known biomarker of OC) was not identified in one of the datasets under investigation [52].

The next step of the investigation involved in-depth analysis of expression of above TFs at different stages of OC. For this, we compared the expression levels of genes in cancerous tissues categorized as stage IA, IC and IIIC [54]. Figure 4 explains that the expression of TFAP2A, VDR, TFAP2G and E2F3 genes is higher in early stages (stages IA and IC) as well as advanced stage (IIIC) of the OC as compared to the normal tissue. The same applies to the gene expression levels of CA125. This analysis clearly shows that the proposed TFs can be tested as diagnostic biomarkers for OC in detailed laboratory investigations. Other important information linked to some of the TFs that are proposed as biomarkers is their detectable expression in blood. For example, VDR [55], E2F3 [56] and CREB1 [57] can be easily detected in the blood and blood-based diagnostic assays can be easily developed to measure these TFs if validated as biomarkers of OC. Further experimental investigations can also focus on establishing a link between blood concentration of biomarkers and pathological state of the ovary in OC.

Since the biomarkers identified were found to be promising we further looked at the pathways involved/associated with the 17 TFs. The current data demonstrates that the TFs identified as biomarkers are associated with 
Table 2 Validation of biomarkers based on over-expression in published OC microarray datasets available in databases such as CleanEx and Oncomine

\begin{tabular}{lcccc}
\hline & TFs regulating all $\mathbf{3 2 3}$ genes & TFs regulating Estrogen controlled sub-set of genes \\
\hline \multicolumn{2}{c}{ Out of top ranked $\mathbf{1 7}$} & Out of top ranked 17 \\
\hline CleanEX & \multicolumn{1}{c}{} & & $\mathbf{2}$ \\
\hline Oncomine & Fold change $>=2$ & Fold change $>=1.5$ & Fold change $>=2$ & Fold change $>=1.5$ \\
\hline TFs found & 8 & 10 & 6 & 6 \\
\hline Total over-expressed TFs & $\mathbf{9}$ & $\mathbf{1 1}$ & $\mathbf{8}$ & $\mathbf{8}$ \\
\hline
\end{tabular}

cell cycle and cell signaling pathways that were known to have a significant role in affecting cancer at the initiation stage as well as metastatic spread (additional files 8 and 9). Functional categorization as identified using Gene/Gene Set overlap matrix with 17 TFs identified as biomarkers for full set of OC genes (additional file 8) showed overlap which were prominent for cell cycle associated TFs based on data from E2F1, E2F3-4 and TFDP-1 genes. Estrogen- controlled sub-set of $\mathrm{OC}$ genes were also found to be under the influence of cell cycle associated TFs (additional file 9). Overlap pattern indicates that TFs associated with $\mathrm{G} 1$ to $\mathrm{S}$ phase transition were found to be mostly affected. Transcription factors DP-1 and E2F1 showed similar overlap patterns in the gene sets since TFDP-1 encodes a member of a family of transcription factors that heterodimerize with E2F proteins to enhance their DNA-binding activity and promote transcription from E2F target genes.

We propose that the TFs identified as biomarkers in the current study may have a prominent role associated with genes involved in progression of OC. Our previous study has identified E2F5 (a member of E2F family of TFs) as a potential marker for detecting the malignancy associated with $\mathrm{OC}$ [6].

\section{(d) Physiological significance of predicted TFs}

Here we describe the significance of TFs identified in our current study and relevance of these TFs to $\mathrm{OC}$ as reported by recent studies. There is growing evidence that deregulation of cell cycle associated transcription factors of E2F family (E2F1 to E2F8) is causatively involved in the patho-physiology of various tumors.
Proliferation-promoting E2F transcription factors, E2F1 and especially E2F2 play a pivotal role in tumor biology of $\mathrm{OC}$ and may be candidates for specific therapeutic targets [58]. In the same study, authors demonstrate higher expression of E2F family of TFs in 77 ovarian cancer samples except E2F6. They also suggested that expression levels of E2F1 and E2F2 are associated with highly malignant and fast growing tumors. Deregulation of both proliferation-promoting and proliferation-inhibiting E2F TFs and their cross-talk were reported to influence the clinical outcome Therefore; appearance of three members of E2F family in our top ranked list also warrants further investigations to explore biomarker potential of these TFs. CREB1 is another TF identified in the current study is associated with MMP-2 transcription, which is involved in pro-metastatic function along with TG-2. EP-300 protein is a histone acetyltransferase and regulates transcription via chromatin remodeling. It is found that this protein regulates important cellular processes such as cell proliferation and differentiation. The putative tumour suppressor gene EP300 is located on the region 22q13 that shows frequent loss of heterozygosity $(\mathrm{LOH})$ in colon, breast and ovarian cancers. In epithelial cancers, EP300 is mutated and provide the evidence that it behaves as a classical tumor-suppressor gene [59]. Higher immunoreactivity of vitamin D receptor (VDR) was found in breast, ovarian and cervical carcinomas as compared to normal corresponding tissues [60]. Recently, the VDR polymorphism FokI was shown to be associated with susceptibility to OC. These results suggest that the VDR polymorphisms from the FokI genotype may be

Table 3 The average fold-change in expression of TFs identified as potential biomarkers in the present study and CA 125 (a known OC biomarker) in ovarian serous adenocarcinoma tissues samples in comparison to normal tissue based on three OC pre-analyzed datasets available in Oncomine

\begin{tabular}{lccc}
\hline Biomarkers & Lu et al., 2004 [54] & Hendrix et al., 2006 [53] & Adib et al., 2004 [52] \\
\hline TFAP2A & 1.99 & 1.57 & 1.21 \\
\hline VDR & 1.66 & 1.85 & 1.22 \\
\hline TFAP2G & 1.33 & 1.18 & 2.18 \\
\hline E2F3 & 1.84 & 2.97 & 2.65 \\
\hline CA 125 & 3.33 & - \\
\hline
\end{tabular}

The values in the table represent the fold-change of expression of genes with p-values $<0.05$ and the gene ranks in top $10 \%$ (pre-calculated in Oncomine). 


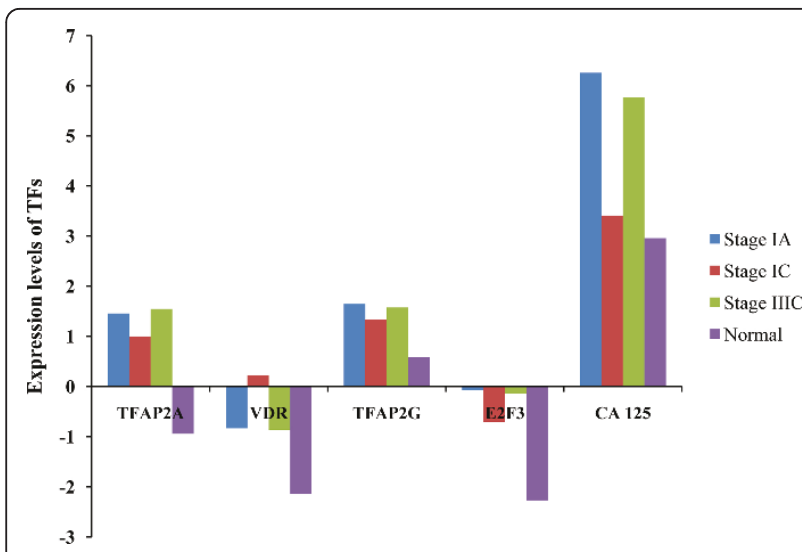

Figure 4 The comparison of the expression levels of TFs identified as potential biomarkers in the present study and CA 125 (a known OC biomarker) in randomly chosen ovarian serous adenocarcinoma tissue samples categorized at stages IA, IC and IIIC in comparison to normal tissue based on OC pre-analyzed dataset of study by Lu et al., 2004 [54]available in Oncomine.

associated with improved prognosis of patients with EOC [61]. The PAX5 gene belongs to the paired box (PAX) family of transcription factors. PAX5 has been reported to be present in ovarian tumor tissues and plasma [62]. Elk-1 has been associated with OC progression [63].

One should observe the following limitations of our methods. We have several constraining factors involved. First, we limited consideration of computational analysis of transcription regulation to promoters of limited size proximal to $5^{\prime}$ gene ends. While there is no method to accurately pinpoint boundaries of promoter regions, we used $1000 \mathrm{bp}$ upstream and $200 \mathrm{bp}$ downstream of TSS as an approximation of the actual promoters. Second, a lot of gene control is exerted from the remote control regions such as enhancers and silencers. Since we had no means to determine these regions for individual genes we did not consider such regulatory effects. Thirdly, we limited our consideration to TFs that fall above $80^{\text {th }}$ percentile regarding the number of genes they potentially regulate in $\mathrm{OC}$ gene set we considered. Finally, the set of genes implicated in OC that we used is by no means complete. However, in spite all these practical odds, the method produced results that are in high concordance with the known biological facts (a number of the top ranked TF are either already known diagnostic markers of represent previously proposed markers for diagnosis of $\mathrm{OC}$ ) and also implies some of the new diagnostic markers for OC.

\section{Conclusions}

The analysis presented here has generated potential molecular targets for evaluation as possible players in the causation or characterization of OC. The ranked lists of TFs can be used to prioritize putative biomarkers based on their potential to regulate a large number of genes implicated in OC. The analysis shown here has provided deeper insights into the transcriptional regulation of many genes involved in OC. We highlight that this is an understudied field and the use of bioinformatics tools could enhance our insights into the underlying genetic mechanism of OC. This study has provided a list of potential target genes that could be tested in the laboratory and ultimately be targets for therapeutic treatments.

\section{Methods}

\section{Outline of methodology}

The study identified several genetic targets for evaluation as potential diagnostic markers of OC. The promoters of 323 genes implicated in OC [64] were screened for EREs (estrogen response elements). To identify the genes experimentally validated as estrogen responsive, we cross-checked against two databases of documented estrogen-responsive genes, namely KBERG [32] and ERTargetDB [33], neither of which is comprehensive. We divided the genes into four groups: 1) those having predicted EREs and experimental evidence of estrogen control; 2) have predicted EREs but no experimental evidence of estrogen control, 3) have no predicted EREs but have experimental evidence of estrogen control, and 4) neither predicted EREs nor experimental evidence of estrogen control. We then predicted TFBSs (Transcription factor binding sites) on the promoters of OC genes so as to provide a link between genes and TFs that regulate them. For this purpose the Transfac Professional database ver. 11.4 [65] was used. The TFBSs were mapped to the promoters of OC genes using the Match program [66] of the Transfac suite. The enrichment of the mapped TFBSs in the target promoter set relative to a background set was determined. The enriched TFBSs were associated with TFs that are predicted to bind them. In this way we established a link between the target genes and the TFs that regulate their expression. This data was used to reconstruct parts of the transcription regulatory networks of relevance to OC. These networks were analyzed to identify crucial regulators of gene expression, opening the way to identify potential biomarkers that characterize OC gene transcription.

\section{Identification of genes implicated in $\mathrm{OC}$}

The genes implicated in $\mathrm{OC}$ were determined as follows. Initially a list of 900 genes was collected from available online resources like Cancer Gene Census [67]http:// www.sanger.ac.uk/genetics/CGP/Census/, GeneCards [68]http://www.genecards.org/index.shtml, SymAtlas [69], OMIM [70] (Online Mendelian Inheritance in 
Man, 2007) http://www.ncbi.nlm.nih.gov, Ovarian Kaleidoscope Database [71]http://ovary.stanford.edu, Entrez Gene [72]http://www.ncbi.nlm.nih.gov/sites/entrez?db=gene and GenAtlas [73]http://www.genatlas.org/. This list was reduced to 379 by biologists after carefully going through the relevant literature. The genes included in the study were filtered through a strict criterion that the gene expression must be experimentally confirmed in either OC tissue using one of the techniques such as: RT-PCR, immunohistochemistry, western blotting or FISH (Fluorescent In Situ Hybridization). If a gene is documented as having an OC linked SNP, it was also included. The collection of these 379 genes can be explored using DDOC database http://apps.sanbi.ac.za/ddoc/[64].

\section{Promoter sets}

The promoter sequences determined as a region covering $[-1000,+200]$ relative to the transcription start sites (TSS) at the 5' ends of genes were extracted from the Fantom3 dataset $[74,75]$. The promoters were extracted for 323 Entrez IDs corresponding to the OC genes. As a background set, we used a set of selected 11,000 sequences of length 1200 bases from human genome.

\section{Prediction of Estrogen Response Elements (EREs)}

The tool Dragon ERE Finder [76] version 6.0 http:// apps.sanbi.ac.za/ere/index.php was used to predict the EREs on the promoters.

\section{Matching to genes from KBERG database}

The OC genes were cross-checked against all the 1516 experimentally confirmed estrogen-responsive genes in the KBERG database [32].

\section{Matching to genes in ERTargetDB database}

The OC genes were cross-checked against the ERTargetDB [33], http://bioinformatics.wistar.upenn.edu/ ERTargetDB, which contains:- (a) 40 genes with 48 experimentally verified ERE direct binding sites and 11 experimentally verified ERE tethering sites; (b) 42 genes identified via ChIP-on-chip assay for estrogen binding (c) 355 genes from gene expression microarrays; (d) 2659 computationally predicted genes.

\section{Transcription Factor binding sites (TFBSs) mapping}

TFBSs were mapped to promoter sequences using all mammalian matrix models of TFBSs contained in the TRANSFAC Professional database v.11.4 [65]. For this purpose, we used the Match program with a minFP profile for the threshold of the matrix models since the minFP profile contains the optimized threshold values for the core and matrix scores [66] that provide minimum presence of false positive predictions in the predicted TFBS set.

\section{Enrichment of TFBSs patterns and edge identification} Enrichment of TFBSs found in the target set was determined using the method from $[77,78]$. The mapped TFBSs were ranked based on their over-representation index (ORI) [77] and those that were sufficiently enriched (ORI $>=2$, as determined by [77]) were used for further analysis. This step produced links between the target genes and TFs that regulate their expression and represent an edge for network reconstruction.

\section{Network reconstruction and identification of biomarkers}

The genes were clustered again based on the promoter content (presence of TFBSs) and this allowed us to identify the network of genes potentially regulated by individual TFs or their combinations. The identified TFs were ranked based on the number of genes they regulate above $80^{\text {th }}$ percentile. Finally, a list of ranked TFs was generated.

\section{Functional and pathway analyses of network genes}

After identification of networks of genes, next step was to classify these genes based upon their functional properties. We used the existing gene ontology (GO) annotation [79] information for the genes. GO term analysis was performed for all the genes using DAVID (The Database for Annotation, Visualization and Integrated Discovery) version 2.0 [80] and transcripts were clustered based on their functional annotations at GO level 4. This helped us to identify the functional clustering of the genes and their role as a group in various biological processes.

Functional analysis is very useful but provides limited information regarding the involvement of genes in specific pathways. The gene lists were mapped to KEGG [81] pathways using DAVID. Mapping of target genes to KEGG helped us to identify pathways critical to the etiology and development of OC.

The above described methodology enabled us to identify networks of genes potentially regulated by similar TFs and their possible roles in etiology and development of $\mathrm{OC}$, opening a way for insights that could lead to the identification of potential diagnostic markers of OC.

\section{Additional material}

Additional file 1: Functional analysis of all four groups of genes using DAVID. Pathway analysis performed on all four groups of genes using DAVID.

Additional file 2: Distribution of TFBSs and EREs in promoters of genes that contain predicted EREs. Details of gene IDs and positions on promoters where TFBSs and EREs were predicted to have binding sites.

Additional file 3: TFs common and unique to gene groups 1 and 3 This file contains list of genes that were found to be common and unique to groups 1 and 3 . 
Additional file 4: TFs common and unique to gene groups 'experimentally controlled genes' and 3 . This file contains list of genes that were found to be common and unique to groups 3 and 'experimentally controlled genes'.

\section{Additional file 5: TFs common and unique to gene groups} 'experimentally controlled genes' and 4 . This file contains list of genes that were found to be common and unique to groups 4 and 'experimentally controlled genes'.

Additional file 6: TFs with ranks for a set of $\mathbf{3 2 3}$ genes. The list of all TFs with ranks based on number of genes they potentially control from full set of 323 genes.

Additional file 7: Details of primary source of the data for partly validating the biomarkers using CleanEx database. Description of previously published study material that was used for partial validation of biomarkers identified in the current study.

Additional file 8: Functional categorization as identified using Gene/Gene Set overlap matrix with 17 TFs identified as biomarkers for full set of $O C$ genes. The graphical view is a matrix of collections of gene sets, where each colored entry indicates that the two gene sets have a statistically significant overlap. Overlap between gene and gene sets were prominent for cell cycle associated TFs as observed for the first two rows from E2F1, E2F3-4 and TFDP-1 genes). Functional relevance of top ranked 17 biomarkers identified for full set of 323 OC genes in the present investigation

Additional file 9: Functional categorization as identified using Gene/Gene Set overlap matrix with 17 TFs identified as biomarkers for estrogen-controlled sub-set of $\mathbf{7 7}$ OC genes. The graphical view is a matrix of collections of gene sets, where each colored entry indicates that the two gene sets have a statistically significant overlap. Overlap between gene and gene sets were prominent for cell cycle associated TFs. Functional relevance of top ranked 17 biomarkers identified for estrogen-controlled sub-set of $77 \mathrm{OC}$ genes in the present investigation.

\section{Abbreviations}

OC: Ovarian cancer; ERa: estrogen receptor alpha; ERs: estrogen receptors; HREs: Hormone-Response Elements; EREs: estrogen response elements; AP: activator protein; NF-B: nerve factor- $\beta$; TFs: Transcription factors; TFBSs: TF binding sites; TSSs: transcription start sites; PTEN : phosphatase and tensin homolog deleted on chromosome 10; LOH: loss of heterozygosity; VDR vitamin D receptor; FISH: Fluorescent In Situ Hybridization; ORI: overrepresentation index; GO: gene ontology.

\section{Acknowledgements}

None

\section{Author details}

${ }^{1}$ Computational Bioscience Research Center, King Abdullah University of Science and Technology, Thuwal 23955-6900, Kingdom of Saudi Arabia. ${ }^{2}$ Centre for Excellence in Genomic Medicine Research, King Abdul Aziz University, PO. Box 80216, Jeddah 21589, Kingdom of Saudi Arabia. ${ }^{3}$ Diagnostic Biomarker Discovery Laboratory, Department of Obstetrics and Gynaecology, Yong Loo Lin School of Medicine, National University Health System, 5 Lower Kent Ridge Road, 119074, Singapore.

\section{Authors' contributions}

MK and VBB conceptualized and designed the study, performed analysis, interpreted data, wrote and revised the manuscript. CMP and SS analyzed the data. KN and MC participated in validation of the data and assessed the biological significance of the predictions. All authors have given final approval of the version to be published.

\section{Conflicts of interest}

The authors declare that they have no competing interests.

Received: 16 April 2011 Accepted: 19 September 2011

Published: 19 September 2011
References

1. Jemal A, Tiwari RC, Murray T, Ghafoor A, Samuels A, Ward E, Feuer EJ, Thun MJ: Cancer statistics, 2004. CA Cancer J Clin 2004, 54:8-29.

2. Agarwal R, Kaye SB: Ovarian cancer: strategies for overcoming resistance to chemotherapy. NatRevCancer 2003, 3:502-516.

3. Sasaroli D, Coukos G, Scholler N: Beyond CA125: the coming of age of ovarian cancer biomarkers. Are we there yet? Biomark Med 2009, 3:275-288

4. Das PM, Bast RC Jr: Early detection of ovarian cancer. Biomark Med 2008, 2:291-303

5. Zhang Z, Chan DW: The road from discovery to clinical diagnostics: lessons learned from the first FDA-cleared in vitro diagnostic multivariate index assay of proteomic biomarkers. Cancer Epidemiol Biomarkers Prev 2010, 19:2995-2999.

6. Kothandaraman N, Bajic V, Brendan P, Huak C, Keow P, Razvi K, SaltoTellez M, Choolani M: E2F5 status significantly improves malignancy diagnosis of epithelial ovarian cancer. BMC cancer 2010, 10:64

7. Crijns A, Fehrmann R, De Jong S, Gerbens F, Meersma G, Klip H, Hollema H, Hofstra R, Te Meerman G, de Vries E: Survival-related profile, pathways, and transcription factors in ovarian cancer. PLoS Med 2009, 6:e1000024.

8. Libermann $T$, Zerbini L: Targeting transcription factors for cancer gene therapy. Current gene therapy 2006, 6:17-34.

9. Turner D, Watson D: ETS transcription factors: oncogenes and tumo suppressor genes as therapeutic targets for prostate cancer. Expert Review of Anticancer Therapy 2008, 8:33-42.

10. Koyanagi K, O'Day S, Gonzalez R, Lewis K, Robinson W, Amatruda T, Kuo C, Wang $\mathrm{H}$, Milford $\mathrm{R}$, Morton D: Microphthalmia transcription factor as a molecular marker for circulating tumor cell detection in blood of melanoma patients. Clinical cancer research 2006, 12:1137.

11. Berghmans T, Paesmans M, Mascaux C, Martin B, Meert A, Haller A, Lafitte J, Sculier J: Thyroid transcription factor 1-a new prognostic factor in lung cancer: a meta-analysis. Annals of oncology 2006, 17:1673.

12. Redell M, Tweardy D: Targeting transcription factors for cancer therapy. Current pharmaceutical design 2005, 11:2873-2887.

13. Pipinikas C, Nair S, FENSKE C: Measurement of blood E2F3 mRNA in prostate cancer by quantitative RT-PCR: a preliminary study. Biomarkers 2007, 12:541-557

14. Pipinikas CP, Carter ND, Corbishley CM, Fenske CD: HIF-1alpha mRNA gene expression levels in improved diagnosis of early stages of prostate cancer. Biomarkers 2008, 13:680-691.

15. Aranda A, Pascual A: Nuclear hormone receptors and gene expression. Physiol Rev 2001, 81:1269-1304.

16. Jeon YT, Kim YB, Park SY, Kim JW, Park NH, Kang SB, Song YS: Gonadotropin-releasing Hormone Receptor Expression in Endometria Cancer. IntJ GynecolPathol 2008.

17. Mao HL, Zhu ZQ, Chen CD: The androgen receptor in hormone-refractory prostate cancer. Asian J Androl 2008.

18. Shi WF, Bartlett JS: Estrogen plays a critical role in AAV2-mediated gene transfer in ovarian cancer. Acta PharmacolSin 2008, 29:1440-1450.

19. Suzuki F, Akahira JI, Miura I, Suzuki T, Ito K, Hayashi SI, Sasano H, Yaegashi N: Loss of estrogen receptor beta isoform expression and its correlation with aberrant DNA methylation of the 5 '-untranslated region in human epithelial ovarian carcinoma. Cancer Sci 2008

20. Walker G, MacLeod K, Williams AR, Cameron DA, Smyth JF, Langdon SP: Estrogen-regulated gene expression predicts response to endocrine therapy in patients with ovarian cancer. GynecolOncol 2007, 106:461-468.

21. Bowman A, Gabra H, Langdon SP, Lessells A, Stewart M, Young A, Smyth JF: CA125 response is associated with estrogen receptor expression in a phase II trial of letrozole in ovarian cancer: identification of an endocrine-sensitive subgroup. ClinCancer Res 2002, 8:2233-2239.

22. Papadimitriou CA, Markaki S, Siapkaras J, Vlachos G, Efstathiou E, Grimani I, Hamilos G, Zorzou M, Dimopoulos MA: Hormonal therapy with letrozole for relapsed epithelial ovarian cancer. Long-term results of a phase II study. Oncology 2004, 66:112-117.

23. Smyth JF, Gourley C, Walker G, MacKean MJ, Stevenson A, Williams AR, Nafussi AA, Rye T, Rye R, Stewart M, et al: Antiestrogen therapy is active in selected ovarian cancer cases: the use of letrozole in estrogen receptorpositive patients. ClinCancer Res 2007, 13:3617-3622.

24. Lacey JV Jr, Mink PJ, Lubin JH, Sherman ME, Troisi R, Hartge P, Schatzkin A Schairer C: Menopausal hormone replacement therapy and risk of ovarian cancer. JAMA 2002, 288:334-341. 
25. Garcia-Velasco A, Mendiola C, Sanchez-Munoz A, Ballestin C, Colomer R, Cortes-Funes H: Prognostic value of hormonal receptors, p53, ki67 and HER2/neu expression in epithelial ovarian carcinoma. ClinTrans/Oncol 2008, 10:367-371.

26. Eliassen $\mathrm{AH}$, Hankinson SE: Endogenous hormone levels and risk of breast, endometrial and ovarian cancers: prospective studies. AdvExpMedBiol 2008, 630:148-165

27. Musumarra G, Barresi V, Condorelli DF, Scire S: A bioinformatic approach to the identification of candidate genes for the development of new cancer diagnostics. Biol Chem 2003, 384:321-327.

28. Kaur M, Schmeier S, Macpherson CR, Hofmann O, Hide WA, Taylor S, Willcox N, Bajic VB: Prioritizing genes of potential relevance to diseases affected by sex hormones: an example of Myasthenia Gravis. BMC Genomics 2008, 9:481.

29. Kun Y, How LC, Hoon TP, Bajic VB, Lam TS, Aggarwal A, Sze HG, Bok WS, Yin WC, Tan P: Classifying the estrogen receptor status of breast cancers by expression profiles reveals a poor prognosis subpopulation exhibiting high expression of the ERBB2 receptor. HumMolGenet 2003, 12:3245-3258.

30. Lin CY, Strom A, Vega VB, Kong SL, Yeo AL, Thomsen JS, Chan WC, Doray B, Bangarusamy DK, Ramasamy A, et al: Discovery of estrogen receptor alpha target genes and response elements in breast tumor cells. Genome Biol 2004, 5:R66.

31. Tang S, Tan SL, Ramadoss SK, Kumar AP, Tang MH, Bajic VB: Computational method for discovery of estrogen responsive genes. Nucleic Acids Res 2004, 32:6212-6217.

32. Tang S, Zhang Z, Tan SL, Tang MH, Kumar AP, Ramadoss SK, Bajic VB: KBERG: KnowledgeBase for Estrogen Responsive Genes. Nucleic Acids Res 2007, 35:D732-D736.

33. Jin VX, Sun H, Pohar TT, Liyanarachchi S, Palaniswamy SK, Huang TH, Davuluri RV: ERTargetDB: an integral information resource of transcription regulation of estrogen receptor target genes. $J$ MolEndocrinol 2005, 35:225-230.

34. Wahli W, Martinez E, Cort'esy B, Cardinaux J: cis-and trans-acting elements of the estrogen-regulated vitellogenin gene B1 of Xenopus laevis. Journal of steroid biochemistry 1989, 34:17-32.

35. Carroll JS, Meyer CA, Song J, Li W, Geistlinger TR, Eeckhoute J, Brodsky AS, Keeton EK, Fertuck KC, Hall GF, et al: Genome-wide analysis of estrogen receptor binding sites. NatGenet 2006, 38:1289-1297.

36. Marilley D, Robyr D, Schild-Poulter C, Wahli W: Regulation of the vitellogenin gene B1 promoter after transfer into hepatocytes in primary cultures. Molecular and cellular endocrinology 1998, 141:79-93.

37. Sica G, Vazquez MF, Altorki N, Port J, Lee PC, Liu Y, Hyjek E, Saqi A: PAX-5 expression in pulmonary neuroendocrine neoplasms: its usefulness in surgical and fine-needle aspiration biopsy specimens. AmJ ClinPathol 2008, 129:556-562.

38. Jensen KC, Higgins JP, Montgomery K, Kaygusuz G, van de RM, Natkunam $Y$ : The utility of PAX5 immunohistochemistry in the diagnosis of undifferentiated malignant neoplasms. ModPathol 2007, 20:871-877.

39. Provenzano MJ, Yu L, Hitchler MJ, Fitzgerald MP, Robinson RA, Wayne S, Ver MM, Domann FE: AP-2 participates in the transcriptional control of the amyloid precursor protein (APP) gene in oral squamous cell carcinoma. ExpMolPathol 2007, 83:277-282.

40. Li M, Wang Y, Hung MC, Kannan P: Inefficient proteasomal-degradation pathway stabilizes AP-2alpha and activates HER-2/neu gene in breast cancer. IntJ Cancer 2006, 118:802-811.

41. Bidus MA, Risinger Jl, Chandramouli GV, Dainty LA, Litzi TJ, Berchuck A, Barrett JC, Maxwell GL: Prediction of lymph node metastasis in patients with endometrioid endometrial cancer using expression microarray. ClinCancer Res 2006, 12:83-88

42. Feng J, Qian H, Tian Y: [Application of multi-tumor markers in ovarian carcinoma]. Zhonghua Fu Chan KeZa Zhi 1998, 33:284-286.

43. Dong $L$, Cui $H$, Li XP, Sun LF, Chang XH, Liang XD, Zhu HL: Clinical value of serum CA19-9, CA125 and CP2 in mucinous ovarian tumor: a retrospective study of 273 patients. Zhonghua Fu Chan KeZa Zhi 2008, 43:5-8.

44. Previdi S, Malek A, Albertini V, Riva C, Capella C, Broggini M, Carbone GM, Rohr J, Catapano CV: Inhibition of Sp1-dependent transcription and antitumor activity of the new aureolic acid analogues mithramycin SDK and SK in human ovarian cancer xenografts. Gynecol Oncol 2010, 118:182-188.
45. Khan S, Wu F, Liu S, Wu Q, Safe S: Role of specificity protein transcription factors in estrogen-induced gene expression in MCF-7 breast cancer cells. J MolEndocrinol 2007, 39:289-304.

46. Davidson B, Hadar R, Schlossberg A, Sternlicht T, Slipicevic A, Skrede M, Risberg B, Florenes VA, Kopolovic J, Reich R: Expression and clinical role of DJ-1, a negative regulator of PTEN, in ovarian carcinoma. HumPathol 2008, 39:87-95.

47. Batistuzzo dM, Krey G, Hihi AK, Wahli W: Functional interactions between the estrogen receptor and the transcription activator $\mathrm{Sp} 1$ regulate the estrogen-dependent transcriptional activity of the vitellogenin A1 io promoter. J Biol Chem 1997, 272:18250-18260.

48. Welsh JB, Zarrinkar PP, Sapinoso LM, Kern SG, Behling CA, Monk BJ, Lockhart DJ, Burger RA, Hampton GM: Analysis of gene expression profiles in normal and neoplastic ovarian tissue samples identifies candidate molecular markers of epithelial ovarian cancer. Proc Natl Acad Sci USA 2001, 98:1176-1181.

49. Praz $V$, Jagannathan $V$, Bucher P: CleanEx: a database of heterogeneous gene expression data based on a consistent gene nomenclature. Nucleic Acids Res 2004, 32:D542-547.

50. Su Al, Welsh JB, Sapinoso LM, Kern SG, Dimitrov P, Lapp H, Schultz PG, Powell SM, Moskaluk CA, Frierson HF Jr, Hampton GM: Molecular classification of human carcinomas by use of gene expression signatures. Cancer Res 2001, 61:7388-7393.

51. Rhodes D, Yu J, Shanker K, Deshpande N, Varambally R, Ghosh D, Barrette T, Pandey A, Chinnaiyan A: ONCOMINE: a cancer microarray database and integrated data-mining platform. Neoplasia (New York, NY) 2004, 6:1.

52. Adib TR, Henderson S, Perrett C, Hewitt D, Bourmpoulia D, Ledermann J, Boshoff $\mathrm{C}$ : Predicting biomarkers for ovarian cancer using geneexpression microarrays. Br J Cancer 2004, 90:686-692.

53. Hendrix ND, Wu R, Kuick R, Schwartz DR, Fearon ER, Cho KR: Fibroblast growth factor 9 has oncogenic activity and is a downstream target of Wnt signaling in ovarian endometrioid adenocarcinomas. Cancer Res 2006, 66:1354-1362.

54. Lu KH, Patterson AP, Wang L, Marquez RT, Atkinson EN, Baggerly KA, Ramoth LR, Rosen DG, Liu J, Hellstrom I, et al: Selection of potential markers for epithelial ovarian cancer with gene expression arrays and recursive descent partition analysis. Clin Cancer Res 2004, 10:3291-3300.

55. Kreutz M, Andreesen R, Krause SW, Szabo A, Ritz E, Reichel H: 1,25dihydroxyvitamin D3 production and vitamin D3 receptor expression are developmentally regulated during differentiation of human monocytes into macrophages. Blood 1993, 82:1300-1307.

56. Pipinikas CP, Nair SB, Kirby RS, Carter ND, Fenske CD: Measurement of blood E2F3 mRNA in prostate cancer by quantitative RT-PCR: a preliminary study. Biomarkers 2007, 12:541-557.

57. Belzeaux R, Formisano-Treziny C, Loundou A, Boyer L, Gabert J, Samuelian JC, Feron F, Naudin J, Ibrahim EC: Clinical variations modulate patterns of gene expression and define blood biomarkers in major depression. J Psychiatr Res 2010.

58. Reimer D, Sadr S, Wiedemair A, Goebel G, Concin N, Hofstetter G, Marth C, Zeimet AG: Expression of the E2F family of transcription factors and its clinical relevance in ovarian cancer. Ann N Y Acad Sci 2006, 1091:270-281.

59. Gayther S, Batley S, Linger L, Bannister A, Thorpe K, Chin S, Daigo Y, Russell P, Wilson A, Sowter H: Mutations truncating the EP300 acetylase in human cancers. Nature genetics 2000, 24:300-303.

60. Friedrich M, Rafi L, Mitschele T, Tilgen W, Schmidt W, Reichrath J: Analysis of the vitamin D system in cervical carcinomas, breast cancer and ovarian cancer. Recent results in cancer research Fortschritte der Krebsforschung Progrès dans les recherches sur le cancer 2003, 164:239.

61. Tamez S, Norizoe C, Ochiai K, Takahashi D, Shimojima A, Tsutsumi Y, Yanaihara N, Tanaka T, Okamoto A, Urashima M: Vitamin D receptor polymorphisms and prognosis of patients with epithelial ovarian cancer. British journal of cancer 2009.

62. Melnikov A, Scholtens D, Godwin A, Levenson V: Differential methylation profile of ovarian cancer in tissues and plasma. Journal of Molecular Diagnostics 2009, 11:60.

63. Bourguignon L, Gilad E, Rothman K, Peyrollier K: Hyaluronan-CD44 interaction with IQGAP1 promotes Cdc42 and ERK signaling, leading to actin binding, Elk-1/estrogen receptor transcriptional activation, and ovarian cancer progression. Journal of Biological Chemistry 2005, 280:11961. 
64. Kaur M, Radovanovic A, Essack M, Schaefer U, Maqungo M, Kibler T, Schmeier S, Christoffels A, Narasimhan K, Choolani M, Bajic VB: Database for exploration of functional context of genes implicated in ovarian cancer. Nucleic Acids Res 2008.

65. Matys V, Fricke E, Geffers R, Gossling E, Haubrock M, Hehl R, Hornischer K, Karas D, Kel AE, Kel-Margoulis OV, et al: TRANSFAC: transcriptional regulation, from patterns to profiles. Nucleic Acids Res 2003, 31:374-378.

66. Kel AE, Gossling E, Reuter I, Cheremushkin E, Kel-Margoulis OV, Wingender E: MATCH: A tool for searching transcription factor binding sites in DNA sequences. Nucleic Acids Res 2003, 31:3576-3579.

67. Futreal PA, Coin L, Marshall M, Down T, Hubbard T, Wooster R, Rahman N, Stratton MR: A census of human cancer genes. NatRevCancer 2004, 4:177-183.

68. Safran M, Solomon I, Shmueli O, Lapidot M, Shen-Orr S, Adato A, Ben Dor U, Esterman N, Rosen N, Peter I, et al: GeneCards 2002: towards a complete, object-oriented, human gene compendium. Bioinformatics 2002, 18:1542-1543.

69. Su Al, Wiltshire T, Batalov S, Lapp H, Ching KA, Block D, Zhang J, Soden R, Hayakawa M, Kreiman $G$, et al: A gene atlas of the mouse and human protein-encoding transcriptomes. ProcNat/AcadSci USA 2004, 101:6062-6067.

70. Baxevanis AD: Searching Online Mendelian Inheritance in Man (OMIM) for information for genetic loci involved in human disease. CurrProtocHumGenet 2003, Chapter 9, Unit9.

71. Leo CP, Vitt UA, Hsueh AJ: The Ovarian Kaleidoscope database: an online resource for the ovarian research community. Endocrinology 2000, 141:3052-3054.

72. Maglott D, Ostell J, Pruitt KD, Tatusova T: Entrez Gene: gene-centered information at NCBI. Nucleic Acids Res 2007, 35:D26-D31.

73. Frezal J: Genatlas database, genes and development defects. C RAcadSci III 1998, 321:805-817.

74. Carninci P, Kasukawa T, Katayama S, Gough J, Frith MC, Maeda N, Oyama R, Ravasi T, Lenhard B, Wells C, et al: The transcriptional landscape of the mammalian genome. Science 2005, 309:1559-1563.

75. Carninci P, Sandelin A, Lenhard B, Katayama S, Shimokawa K, Ponjavic J, Semple CA, Taylor MS, Engstrom PG, Frith MC, et al: Genome-wide analysis of mammalian promoter architecture and evolution. NatGenet 2006, 38:626-635.

76. Bajic VB, Tan SL, Chong A, Tang S, Strom A, Gustafsson JA, Lin CY, Liu ET: Dragon ERE Finder version 2: A tool for accurate detection and analysis of estrogen response elements in vertebrate genomes. Nucleic Acids Res 2003, 31:3605-3607.

77. Bajic VB, Choudhary V, Hock CK: Content analysis of the core promoter region of human genes. In SilicoBiol 2004, 4:109-125

78. Bajic VB, Tan SL, Christoffels A, Schönbach C, Lipovich L, Yang L, Hofmann O, Kruger A, Hide W, Kai C, et al: Mice and Men: Their Promoter Properties. PLoS Genet 2006, 2:e54.

79. Ashburner M, Ball CA, Blake JA, Botstein D, Butler H, Cherry JM, Davis AP, Dolinski K, Dwight SS, Eppig JT, et al: Gene ontology: tool for the unification of biology. The Gene Ontology Consortium. NatGenet 2000, 25:25-29.

80. Dennis G Jr, Sherman BT, Hosack DA, Yang J, Gao W, Lane HC, Lempicki RA: DAVID: Database for Annotation, Visualization, and Integrated Discovery. Genome Biol 2003, 4:3.

81. Kanehisa M, Goto S: KEGG: kyoto encyclopedia of genes and genomes. Nucleic Acids Res 2000, 28:27-30.

doi:10.1186/1752-0509-5-144

Cite this article as: Kaur et al: In Silico discovery of transcription factors as potential diagnostic biomarkers of ovarian cancer. BMC Systems Biology 2011 5:144.

\section{Submit your next manuscript to BioMed Central and take full advantage of:}

- Convenient online submission

- Thorough peer review

- No space constraints or color figure charges

- Immediate publication on acceptance

- Inclusion in PubMed, CAS, Scopus and Google Scholar

- Research which is freely available for redistribution

Submit your manuscript at www.biomedcentral.com/submit
Biomed Central 